\title{
A Novel Resource Dissemination and Discovery Model for Pervasive Environments Using Mobile Agents
}

\author{
Ebrahim Bagheri, Mahmood Naghibzadeh, and Mohsen Kahani \\ Department of Computing, Ferdowsi University of Mashhad, \\ Mashhad, Iran \\ Eb_ba63@stu-mail.um.ac.ir, naghib@um.ac.ir, \\ kahani@um.ac.ir
}

\begin{abstract}
Pervasive computing has been the new buzz word in the distributed computing field. Vast range of heterogeneous resources including different devices, computational resources and services world wide are virtually summoned under agreed conditions to form a single computational system image [1].In this approach a central controller is needed to coordinate the resources which is called the resource management system (RMS).A typical RMS is responsible for three main jobs including matching, scheduling and executing received requests based on suitable and available resources. Regarding the matching module, resource dissemination and discovery forms the main part. In this article we introduce two new approaches to tackle this problem using the mobile agents. The different strategies are dividend into hierarchical and non-hierarchical models. The performed simulations show a better performance for the hierarchical approach compared with the other model.
\end{abstract}

Keywords: Pervasive Environments, Resource Discovery, Resource Dissemination, Virtual Organization, Mobile Agents.

\section{Introduction}

Ubiquitous computing environment is a virtual unified networked set of heterogeneous computers that share their local resources with one another in order to serve the users needs in transparent way. They can encompass many different administrative domains and include lots of different organizations. Resources are usually categorized into independently managed groups based on some criteria and are called virtual organizations [2]. Theses criteria are usually based on the ownership model.

With the emergence of the mobile computing infrastructure the demand for omnipresent services and information has been more than ever before. The devices used in this model are very resource limited and thus should be able to exploit the outer available resources for their needs and also be able to discover these resources in a very cost inexpensive manner. For this reason many different resource management models have been proposed and implemented. Different models serve different intentions and requirements of a specific environment and based upon the resources available, but what is clear is that the main functionality of the RMS is to accept incoming distributed requests, find the matching machines containing the required resources and allocate the resources to the requests and schedule them. These operations are 
allocate the resources to the requests and schedule them. These operations are mainly executed with the optimized QoS offered [3].

Resource dissemination and discovery form a very important part of the resource management service. Although they can be seen as two separate operations but they are mainly integrated to create much more effective results. Resource dissemination mainly focuses on introducing new resources added to the environment to the other machines available. The current dissemination models can be categorized into a periodic pull or push model and on the other hand an on demand request approach.

Resource discovery aims at finding suitable resources residing on disperse machines to serve the incoming requests. This function can be performed using distributed or centralized queries. Different agent models can also be applied. In this article we introduce two new models of resource dissemination and discovery using mobile agents. The models have been completely explained through the rest of the article and simulation results have been shown. The article is conducted in 5 sections. Section 2 elaborates on the architecture of the environment while section 3 will introduce the algorithms and explain the different parts in an in-depth manner. The simulation results will be shown in section 4 and then the article will be concluded in section 5 .

\section{Environment Architecture}

There are an enormous number of resources lying dispersedly on different machines in a pervasive computing environment. The architecture in which the resources are categorized is of much importance due to scheduling decisions, communication structure, etc that need to be considered [4].

In our approach we assume independent administrative domains to be in charge of a set of resources owned by a specific authority. These domains are called Virtual Organizations (VO). Resources are hence owned by exclusive virtual organizations. In our model, resources belonging to a specific virtual organization can be scattered throughout the environment and so geographical placement of resources doesn't shape the administrative domain but is the ownership model which is used [5]. This model was selected so that service level agreements and the economical models could be effectively created.

The machines form the different parts of a massive network but in the conceptual layer the virtual organizations play the main role by establishing agreements to share their resources, and cooperating in creating a rich computational environment.

\section{Module Structure}

The module created for the algorithm consists of two main parts: Resource Dissemination, and Resource Discovery.

\subsection{Resource Dissemination}

The resource dissemination algorithm is used to introduce new resources added to different machines in the environment to the other machines and virtual organizations [6]. In our resource dissemination approach, every time that a resource is added to a 
machine inside a specific virtual organization, the resource dissemination algorithm broadcasts the static attributes of the added resource to all of the existing machines inside the same virtual organization. In this phase, every machine residing on the same virtual organization as the machine owning the new resource will be aware of the new resource. To inform other virtual organizations of the existence of such resource, a message is sent to every virtual organization, declaring the existence of the new resource in the source virtual organization. The message passing theme used here is different from the one used inside the same virtual organization in the way that the machine inside the same virtual organization can exactly spot the resource but the other virtual organizations only have a vague awareness of the resource placement inside the corresponding virtual organization.

\subsection{Resource Discovery}

One of the most important parts of the resource management system is the resource discovery algorithm [7]. We propose a new resource discovery model which utilizes mobile agents for resource discovery. In this regard, two hierarchical and nonhierarchical models have been created.

In the non-hierarchical model, when a resource request arrives at a specific machine the environment knowledge based inside the machine is consulted for matching results. As the machine has only information on the static attributes of the available resources and on the other hand can not exactly locate the position of resources on the other virtual organizations, decision on a suitable resource could not be taken. For this reason a number of mobile agents, according to the number of available resources, are created. The agents are then sent to the attorney of the corresponding virtual organizations. The attorney of a virtual organization is not statically designated but as we have defined is randomly selected every time from the available machines in the destination virtual organization based on network load and originating virtual organization closeness and will avoid bottleneck creation which is anticipated in the case of a static attorney. The attorney will then again consult its knowledge base to exactly locate the resource(s) available in that virtual organization. It will hence create a number of mobile agents which are again as the same number as the available resources, to move to the corresponding machines and assess the dynamic attributes and also evaluate connection parameters. The mobile agents will then return to the attorney and pass back the results. The attorney will select the best resource and introduce it to the waiting agent. The agent will, on its turn, return to the originating machine on the source virtual organization. The mobile agents returned from many different virtual organizations will feedback the information collected and the best resource is then selected. Using the mobile agents for the discovery purpose not only allow us to precisely accumulate the dynamic parameters of a resource such as the cpu load, the length of the printer queue and etc. but it will also enable us to roughly estimate the network conditions and estimate the time to execution of the job. As the resources may suddenly leave or stop functioning, the agents will also update the knowledge base of every machine in the visiting virtual organization resulting in up-to-date machines.

The hierarchical algorithm on the hand uses a cascading model. The source virtual organization is primarily queried for suitable resources to match the request and if suitable results are not found, agents are sent to external virtual organizations to 
discover available resources and gather required information. The decision to which machine has the suitable resource is then taken.

\section{Simulation and Evaluation}

The model was simulated and implemented and fifteen different experiments were conducted. The simulations' test data were chosen from different natures to test algorithm behavior under different conditions.

The first five experiments studied the effect of variance of the number of resources (SVSNDR) while the second five set of simulations focused on the change in the number of nodes (SVDNSR) while the number of resources and virtual organization were left untouched. The last set of conducted simulations focused on the outcome of the virtual organization quantity change (DVSNSR).The resource distributions in the 15 undertaken experiments are shown in figures 1 to 3.For comparison purposes different performance evaluation criteria where introduced which are defined as 1.(Selected/Maximum) Path Ratio: This criterion divides the length of the discovered path to the length of the farthest suitable resource path available in the environment which shows the extent of path length optimization. The path length is calculated based on the hops to reach the destination. (Figure 4). 2. Mean Agent Life Time (MALT): This factor shows on an average basis how long an agent created in the algorithm lives in the environment. 3. Number of Active Agents multiplied by their Average Life Span (SL): This factor introduces the exact amount of environment resource consumption by the agents created in the algorithm since the number of active agents multiplied by their life time will allow us to compute the amount of algorithm activity. 4. Maximum Discovery Time (MXDT): This criterion is equal to the effective resource discovery time. 5. Minimum Discovery Time (MNDT): The exploration time of the fastest agent to return the expected results (Figure 5). The minimum discovery time in both approaches belong to the resource discovery process inside the same virtual organization; therefore the diagram for this criterion in both approaches should be similar. The simulation results show the same fact and hence


Fig. 1. Dynamic change in the number of resources

Fig. 2. Dynamic change in the number of nodes 


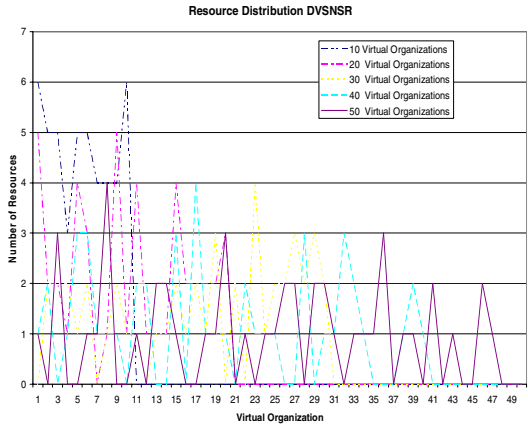

Fig. 3. Dynamic change in the number of Resources

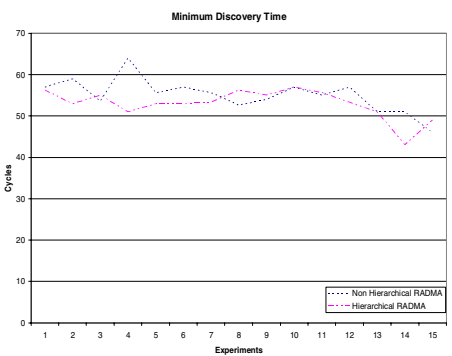

Fig. 5. -Minimum Discovery Time (MNDT)



Fig. 4. (Selected / Maximum) Path Ratio

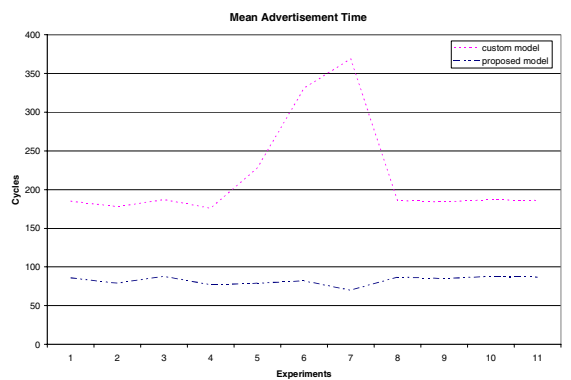

Fig. 6. -Mean Resource Dissemination Time

validate the simulation. On the other hand The MNDT and MXDT diagrams for the hierarchical model were predicted to have similarities caused by the initial originating virtual organization search which is also proved by the simulation outcome.

Although the hierarchical algorithm creates agents with longer life span but the overall environment resource consumption is balanced by creating less mobile agents. The experiments show that the hierarchical algorithm performs much better with higher speed in resource discovery, lower overall system burden on the network traffic and lower environment resource consumption.

The resource dissemination algorithm also shows much better performance compared with the normal resource broadcast model (Figure 6). It is worth noting that the efficiency of this resource dissemination algorithm is enhanced while it is accompanied with resource discovery models explained in this article.

\section{Conclusion}

In this article we have proposed two new models (hierarchical and non-hierarchical) for resource discovery and a complementing resource dissemination algorithm to complete them and provide an efficient module to use inside the resource management system of the pervasive environment management package. Fifteen different experiments were conducted which show a better performance for the hierarchical 
algorithm. This algorithm provides better results along with higher speed in resource discovery, lower overall system burden on the network traffic, and lower environment resource consumption.

\section{References}

1. Kong, Q., Berry, A., "A General Resource Discovery System for Open Distributed Processing", ICODP'95, 1995

2. Foster, I., Kesselman, C., "The Grid: Blueprint for a New Computing Infrastructure " 2nd ed. Morgan Kaufmann, 2004.

3. Krauter, K., Buyya, R., and Maheswaran, M., "A Taxonomy and Survey of Grid Resource Management Systems", Technical Report: University of Manitoba (TR-2000/18) and Monash University (TR-2000/80)

4. Czerwinski, S., Zhao, B., Hodes, T., Joseph, A., Katz, R., "An architecture for a secure service discovery service", Fifth Annual International Conference on Mobile Computing and Networks (MobiCom '99)

5. Foster, I., Kesselman, C., Tuecke, S., "The Anatomy of the Grid Enabling Scalable Virtual Organizations", Intl J. Supercomputer Applications, 2001.

6. Rakotonirainy, A., Groves, G., "Resource Discovery for Pervasive Environments", 4th International Symposium on Distributed Objects and Applications (DOA2002), 2000.

7. McGrath, R., "Discovery and Its Discontents: Discovery Protocols for Ubiquitous computing". Technical Report UIUCDCS-R-99-2132, 2000 